\title{
The use of super-selective uterine artery branch embolization and methotrexate in cervical pregnancy - case reports and literature review
}

\author{
Tomasz P. Oleksik ${ }^{1, A-D \oplus}{ }^{\oplus}$, Kamil Pluta ${ }^{1, B-D \oplus}$, Tadeusz Issat ${ }^{2,1, B-C \oplus}{ }^{\text {, Artur Jakimiuk }}{ }^{3,1, E-F} \oplus$, \\ Waldemar Wierzba ${ }^{4, C, E \odot}$ \\ ${ }^{1}$ Department of Obstetrics and Gynecology, Central Clinical Hospital of the Ministry of Interior and Administration, \\ Warsaw, Poland \\ ${ }^{2}$ Department of Obstetrics and Gynecology, Institute of Mother and Child, Warsaw, Poland \\ ${ }^{3}$ Centre for Reproductive Health, Institute of Mother and Child, Warsaw, Poland \\ ${ }^{4}$ University of Humanites and Economics, Łódź, Satellite Campus in Warsaw, Poland \\ A - Research concept and design, B - Collection and/or assembly of data, C - Data analysis and interpretation, \\ $D$ - Writing the article, E-Critical revision of the article, F- Final approval of article
}

Oleksik TP, Pluta K, Issat T, Jakimiuk A, Wierzba W. The use of super-selective uterine artery branch embolization and methotrexate in cervical pregnancy - case reports and literature review Ann Agric Environ Med. 2021; 28(3): 521-524. doi: 10.26444/aaem/127926

\begin{abstract}
Cervical ectopic pregnancy (CEP) is one of the rarest types of ectopic pregnancy. Early detection of such cases increases the chances of conservative treatment and fertility preservation. Within 6 weeks of each other, 2 women were admitted to the OB/GYN Department with cervical ectopic pregnancies. They were successfully treated with a double dose of methotrexate and super-selective uterine artery branch embolization (SUABE). Taking into account health and economic considerations, for instance the optimal recovery time, and lower costs of operating theater time, laboratory testing and outpatient observation, it seems that safe, minimally invasive, fertility-saving, effective systemic administration of MTX in combination with SUABE, can be a rational alternative to cervical ectopic pregnancy treatment.
\end{abstract}

\section{Key words}

cervical ectopic pregnancy, Methotrexate, super-selective uterine artery branch embolization, uterine artery embolization

\section{INTRODUCTION}

Cervical pregnancies account for less than $1 \%$ of all ectopic pregnancies [1]. When such cases are detected early, patient mortality is significantly reduced. There is still no standard practice in these cases.

Procedures vary between centres of treatment depending on practitioner experience and access to minimally invasive technology. In our department, non-standard procedures have been applied, which are presented in this paper.

\section{CASE REPORTS}

Patient No. 1. A 32-year-old nullipara presented to the emergency room (ER) with vaginal bleeding and lower abdominal pain. Her last menstrual period had been 8 weeks prior to the ER visit. Bimanual vaginal examination revealed a normal size uterus with ballooning of the cervix and a partially open external cervical os with mild active bleeding. Transvaginal ultrasonography showed an empty uterine cavity with the endometrium widened to $10 \mathrm{~mm}$, that both the ovaries and the fallopian tubes were normal, and the presence of a highly vascularized mass ( 34 × $23 \times 30 \mathrm{~mm})$ with an ovalshaped $12 \mathrm{~mm}$ gestational sac in the cervical canal. A blood sample showed a beta-hCG level of $20,760 \mathrm{mIU} / \mathrm{ml}$. Other blood tests were within normal ranges. The patient had no history of uterine surgery or endometrial curettage (Fig. 1A).

Address for correspondence: Artur Jakimiuk, Centre for Reproductive Health, Institute of Mother and Child, Warsaw, Poland

E-mail: jakimiuk@yahoo.com

Received: 02.09.2020; accepted: 28.09.2020; first published: 07.10.2020
Patient No. 2. A 31-year-old nullipara, 6 weeks after IVF treatment, was admitted to ER with mild lower abdominal pain. A vaginal examination showed a closed external cervical os and no bleeding. Transvaginal ultrasonography revealed normal ovaries and fallopian tubes, an empty uterine cavity with the endometrium widened to $9 \mathrm{~mm}$, and a highly vascularized mass $(26 \times 25 \times 15 \mathrm{~mm})$ with an oblong-shaped gestational sac in the cervical canal. The serum level of beta-hCG was $13,600 \mathrm{mIU} / \mathrm{ml}$. Other blood tests were in normal ranges. The patient had no history of uterine surgery or endometrial curettage (Fig. 1 B).

Methodology. Considering the different treatment options and the centre's capabilities, together with the predictable treatment outcomes, both patients were selected for the same procedure, namely, intramuscular administration of methotrexate (MTX) with 2 doses of $50 \mathrm{mg}$ per square meter of the body area at 7-day intervals, which achieved reduced beta-hCG serum values to $494 \mathrm{mIU} / \mathrm{mL}$ in the first patient, and to $4776 \mathrm{mIU} / \mathrm{mL}$ in the second case, without reduced vascularization as revealed by Doppler ultrasonography undertaken on the second day after systemic treatment; and in addition, treatment with super-selective embolization of the pathological uterine arteries branch (SUABE). Both uterine arteries were selected using a catheter and embolized with PVA-700 microspheres. Post-embolization images showed satisfactory obstruction of arterial flow (Fig. 2A, B, C, D). The beta-HCG values trended down in both patients, to $131 \mathrm{mIU} / \mathrm{mL}$ in case 1 and to 2,403 $\mathrm{mIU} / \mathrm{mL}$ in case 2, on day 2 after SUABE without any bleeding.

Having achieved significant reduction of beta-hCG levels and vascularization observed by Doppler ultrasound, the 


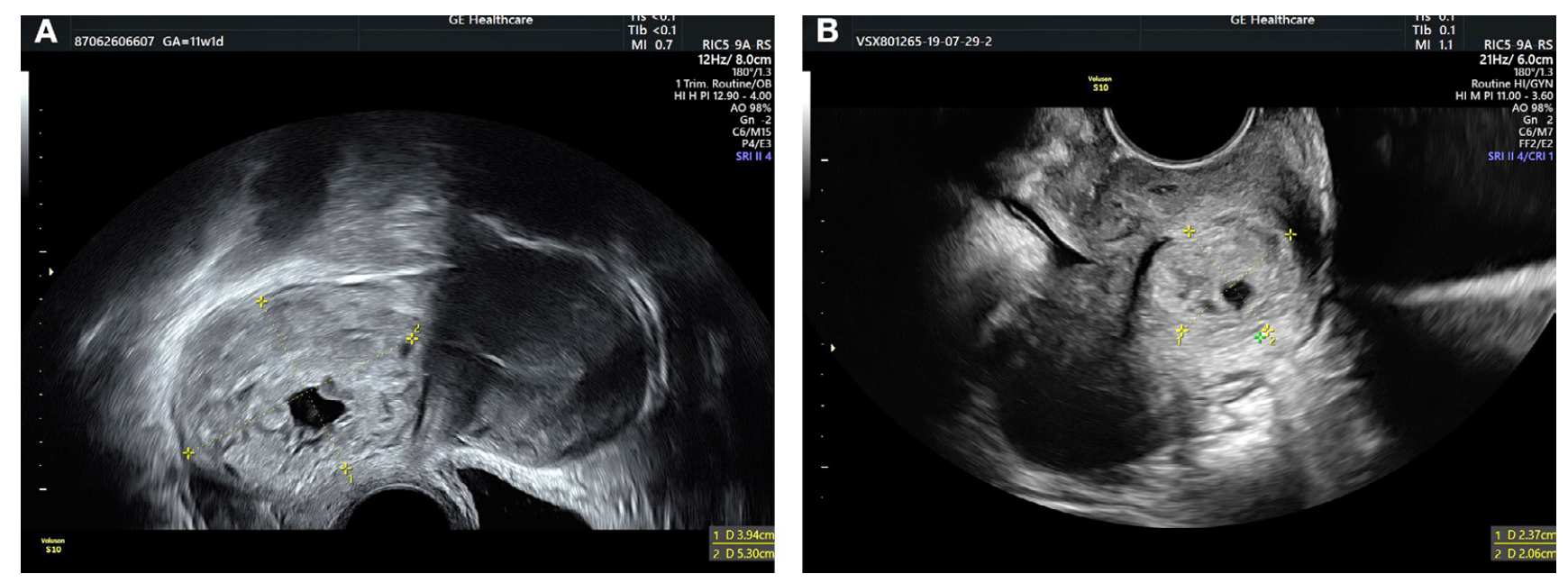

Figure 1. Ultrasonography showing the size and vascularization of a cervix ectopic pregnancy on the day of admission in the first (A) and second patient (B)
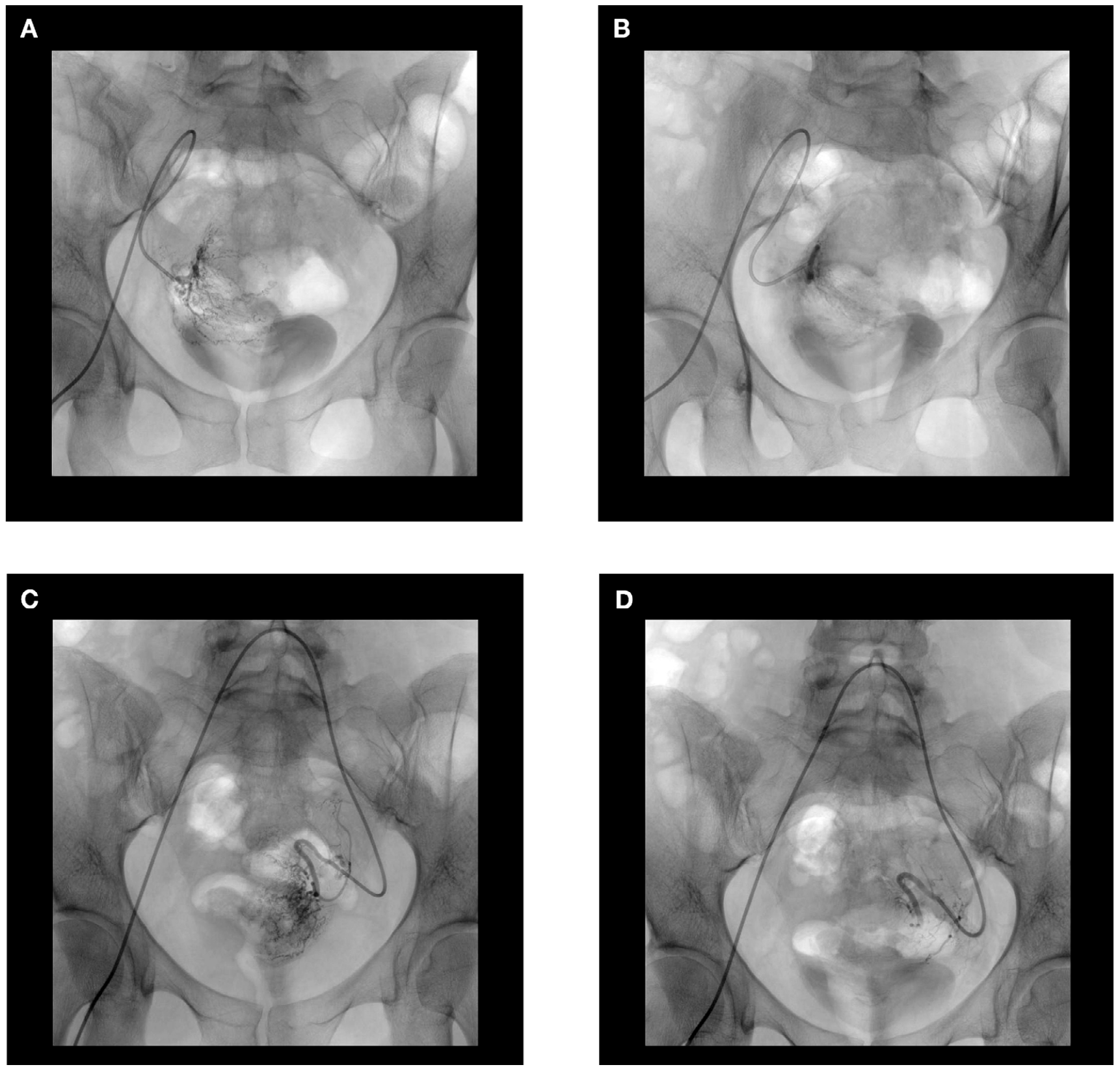

Figure 2. Pre- and post-embolization arteriogram showing the super-selective uterine artery branches embolization (including the cervical component) prior to embolization and after [Pr-embolization $(A, C)$, po-embolization $(B, D)]$ in the first $(A, B)$ and second patient $(C, D)$ 

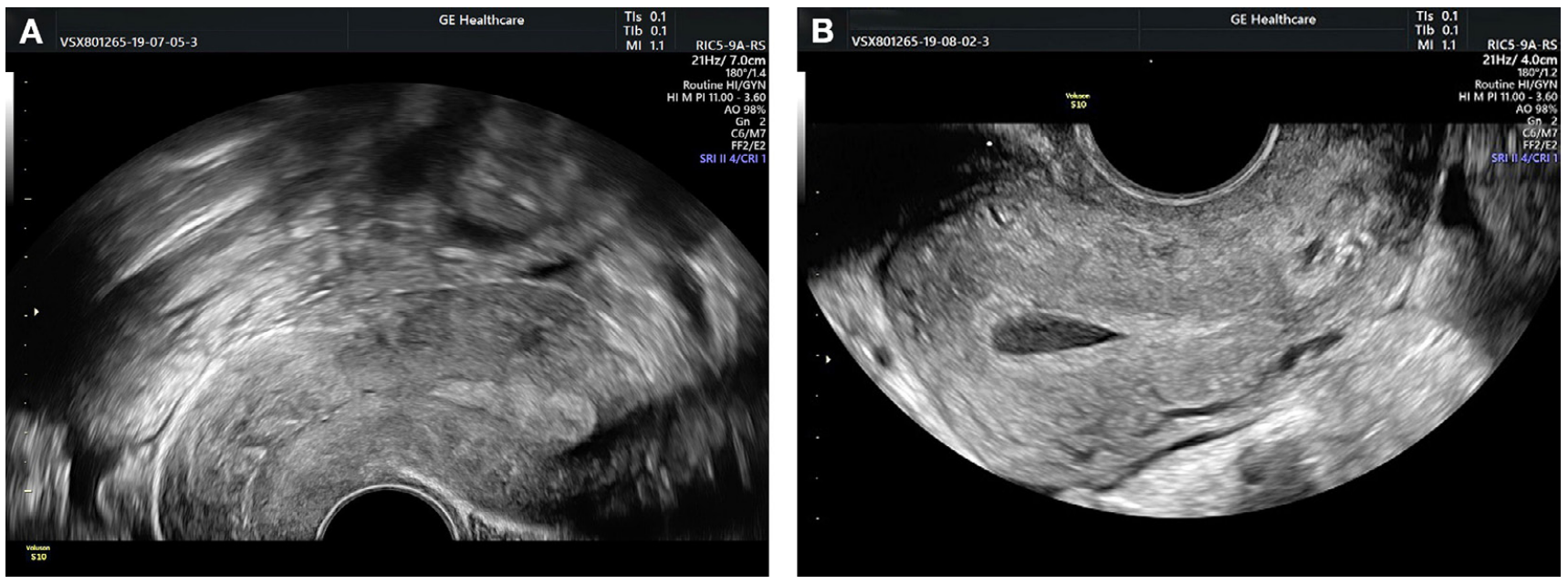

Figure 3. Control ultrasonography showing normal-looking cervix 7 days after the end of treatment in the first (A) and second patient (B)

products of conception were removed under aaesthesia in both patients without complications. One week after discharge from the hospital, their follow-up ultrasound examination showed a normal cervix in each case (Fig. 3A, B).

\section{DISCUSSION}

The literature search revealed no standard procedure or therapeutic options for cases of CEP. CEP is the second rarest form of ectopic pregnancy after abdominal ectopic pregnancy [2], and has a reported incidence of 1/1,000-18,000 pregnancies [3]. Therefore, it is not possible to undertake reliable randomized trials that would clearly determine the most appropriate and safest treatment that decreases the risk of hysterectomy and, consequently, avoids fertility loss in young childless women.

Common risk factors of CEP are prior dilatation and curettage (D and C), which are most commonly undertaken for terminations of pregnancy; prior caesarean section; and in vitro fertilization (IVF) [4]. Diagnosis by sonography is undertaken when the gestational sac is identified as having peritrophoblastic flow or there is a live embryo below the level of a closed internal cervical os within the cervix and with an empty uterine cavity [5]. CEP is associated with significant risk of massive haemorrhage, which is the most common cause of pregnancy-related death during the first trimester [6].

Painless vaginal bleeding is the most common clinical symptom, with only one-third of women presenting with massive haemorrhaging. In 1959, Paalman and McElin [7] offered 5 clinical signs to establish the diagnosis as follows: 1) uterine bleeding without cramping pain after a period of haemenorrhea;

2) softened and disproportionately enlarged cervix equal to or larger than the corporal portion of the uterus (an hourglass-shaped uterus);

3) products of conception entirely confined within, and firmly attached to the endocervix;

4) a snug internal cervical os;

5) a partially opened external cervical os.

Currently, the management of CEP in most patients is conservative, and both Hofmann et al. and Dall et al. consider the most conservative treatment to be effective within the first 12 weeks of pregnancy $[8,9]$. Assessment of the effectiveness of such treatment varies according to the opinions of the above-mentioned research groups, mainly in relation to the depth of trophoblast penetration into the endocervix. The preferred conservative therapy with an effectiveness exceeding $94 \%$, is intramuscular application of one MTX dose of $50 \mathrm{mg} / \mathrm{m}^{2}$ of body area, which is about $1 \mathrm{mg} / \mathrm{kg}$ of body weight [10]. However, the reduction of serum b-hCG levels can take a long time and the risk of massive bleeding, necessitating surgical intervention, remains.

A variety of other conservative treatments have been reported including local injection of methotrexate, KCL, local vasopressin injection, local or systemic prostaglandin, systemic mifepristone, intrauterine irrigation with $3.5 \% \mathrm{H}_{2} \mathrm{O}_{2}$ and uterine artery embolization (UAE) [11].

The presented study demonstrates that super-selective uterine artery branch embolization (SUABE) in combination with a systemic MTX administration could be a prospective alternative in reducing the risk of CEP complications. So far, vessels embolization has been used in gynaecology for chronic pelvic pain relief and palliative care in patients with diagnosed cervical cancer and heavy bleeding. Developments in invasive radiology allow for selective and accurate localization of uterine arteries and pathological vessels within the CEP, which means it can be used in the conservative treatment of such abnormal pregnancies. Takeda et al. also successfully applied bilateral uterine artery embolization (BUAE) and MTX in patients with cervical pregnancy, who, thanks to the applied treatment, avoided significant bleeding $[12,13]$.

Currently, UAE is used worldwide as an effective treatment for CPE in combination with other methods, such as dilation and curettage $[14,15,16]$. If an ectopic pregnancy is not evacuated shortly after embolization, some gestational tissue may remain and, as a result, lead to bleeding; it therefore seems necessary to combine the 2 methods [17].

In several cases, the application of UAE is reported to have resulted in complications, such as pelvic infection, premature ovarian failure and necrosis of the uterine myoma; hence, each CEP case should be considered individually, and patient education should include information about possible complications of the treatment [18]. 


\section{CONCLUSION}

In both cases presented, a combination of methotrexate and super-selective uterine artery branch embolization (SUABE) was used, and the most common and dangerous complications for patients, such as heavy bleeding and hysterectomy, were avoided. Considering the health and economic consideration - for instance, the optimal recovery time, lower costs of operating theater time, laboratory testing and outpatient observation - it seems that safe, minimally invasive, fertilitysaving, and effective systemic administration of MTX in combination with SUABE, can be a rational alternative to cervical ectopic pregnancy treatment.

Undoubtedly, SUABE requires the hospital to have an interventional radiology department, and possibly a more experienced team is needed than that needed to perform BUAE, but if the efficacy of the procedure used in the current presented study are to be confirmed in further studies, it should be considered whether hospitals equipped like the one in the presented cases, should become reference units for the treatment of CEP patients. Further studies comparing this procedure with other treatments are needed.

\section{REFERENCES}

1. Cepni I, Ocal P, Erkan S, et al. Conservative treatment of cervical ectopic pregnancy with transvaginal ultrasound-guided aspiration and single-dose methotrexate. Fertil Steril. 2004; 81(4): 1130-1132. doi: https://doi.org/10.1016/j.fertnstert.2003.09.05

2. Hosni, Mohamed Maged MD, et al. Diagnostic and Therapeutic Dilemmas of Cervical Ectopic Pregnancy. Obstet Gynecol Surv. 2014; 69(5): 261-276. doi: https://doi.org/10.1097/OGX.0000000000000062

3. Taylor JE, Yalcinkaya TM, Akar ME. Successful conservative management of cervical ectopic pregnancy: a case series. Arch Gynecol Obstet. 2011; 283: 1215-1217. doi: https://doi.org/10.1007/s00404-0101529-7

4. Sharma A, Ojha R, Mondal S, et al. Cervical intramural pregnancy: Report of a rare case. Niger Med J. 2013; 54: 271-3. doi: https://doi. org/10.4103/0300-1652.119670
5. Dialani V, Levine D. Ectopic Pregnancy: A Review. Ultrasound Q. 2014; 20(3): 105-117. doi: https://doi.org/10.1097/00013644-20040900000005

6. Segal S, Mercado R, Rivnay B. Ectopic pregnancy early diagnosis markers. Minerva Ginecol. 2010; 62: 49-62.

7. Paalman RJ, McElin TW. Cervical pregnancy. Review of the literature and presentation of cases. Am J Obstet Gynecol. 1959; 77: 1261.

8. Hofmann HM, Urdl W, Höfler H, et al. Cervical pregnancy: case reports and current concepts in diagnosis and treatment. Arch Gynecol Obstet. 1987; 241(1): 63-69. doi: https://doi.org/10.1007/BF00931444

9. Dall P, Pfisterer J, du Bois A, et al. Therapeutic strategies in cervical pregnancy. Eur J Obstet Gynecol Reprod Biol. 1994; 56(3): 195-200. doi: https://doi.org/10.1016/0028-2243(94)90170-8

10. Stovall TG, Ling FW. Single-dose methotrexate: an expanded clinical trial. Am J Obstet Gynecol. 1993; 168(6 Pt 1): 1759-1765. doi: https:// doi.org/10.1016/0002-9378(93)90687-e

11. Hosni MM, Herath RP, Mumtaz R. Diagnostic and therapeutic dilemmas of cervical ectopic pregnancy. Obstet Gynecol Surv. 2014; 69(5): 261-276. doi: https://doi.org/10.1097/OGX.0000000000000062

12. Wozniak S. Chronic pelvic pain. Ann Agric Environ Med. 2016; 23(2): 223-226. doi: https://doi.org/10.5604/12321966.1203880

13. Takeda K, Mackay J, Watts S. Successful Management of Cervical Ectopic Pregnancy with Bilateral Uterine Artery Embolization and Methotrexate. Case Rep Emerg Med. 2018: 9593824. doi: https://doi. org/10.1155/2018/9593824

14. Cosin JA, Bean M, Grow D, et al. The use of methotrexate and arterial embolization to avoid surgery in a case of cervical pregnancy. Fertil Steril. 1997; 67: 1169-71.

15. Vedantham S, Goodwin SC, McLucas B, et al. Uterine artery embolization: an underused method of controlling pelvic hemorrhage. Am J Obstet Gynecol. 1997; 176(4): 938-948. doi: https://doi.org/10.1016/ s0002-9378(97)70624-0

16. Wang Y, Xu B, Dai S, et al. An efficient conservative treatment modality for cervical pregnancy: angiographic uterine artery embolization followed by immediate curettage. Am J Obstet Gynecol. 2011; 204(31): e1-7. doi: https://doi.org/10.1016/j.ajog.2010.08.048

17. Scutiero G, Nappi L, Matteo M, et al. Cervical pregnancy treated by uterine artery embolisation combined with office hysteroscopy. Eur J Obstet Gynecol Reprod Biol. 2013; 166(1): 104-106. doi: https://doi. org/10.1016/j.ejogrb.2012.10.013

18. Yu B, Douglas NC, Guarnaccia MM, et al. Uterine artery embolization as an adjunctive measure to decrease blood loss prior to evacuating a cervical pregnancy. Arch Gynecol Obstet. 2009; 279(5): 721-724. doi: https://doi.org/10.1007/s00404-008-0775-4 